17,16

\title{
Особенности спектров ядерного магнитного резонанса полигидроксилированного фуллерена $\mathrm{C}_{60}(\mathrm{OH})_{n}$
}

\author{
(C) А.С. Мазур ${ }^{1}$, А.Е. Карпунин ${ }^{2}$, О.В. Проскурина ${ }^{3,4}$, В.И. Герасимов ${ }^{2}$, \\ И.В. Плешаков ${ }^{2,4, \uparrow}$, В.В. Матвеев ${ }^{1}$, Ю.И. Кузьмин ${ }^{2,4}$ \\ ${ }^{1}$ Санкт-Петербургский государственный университет, \\ Санкт-Петербург, Россия \\ ${ }^{2}$ Санкт-Петербургский политехнический университет Петра Великого, \\ Санкт-Петербург, Россия \\ ${ }^{3}$ Санкт-Петербургский государственный технологический институт (технический университет), \\ Санкт-Петербург, Россия \\ ${ }^{4}$ Физико-технический институт им. А.Ф. Иофффе РАН, \\ Санкт-Петербург, Россия \\ ฯ E-mail: ivanple@yandex.ru
}

(Поступила в Редакцию 23 января 2018 г.)

Полигидроксилированный фуллерен $\mathrm{C}_{60}(\mathrm{OH})_{n}$ (с оценочным количеством гидроксильных групп $n=38-44)$, синтезированный из чистого фуллерена смешением бензольного раствора $\mathrm{C}_{60}$ с водным раствором $\mathrm{NaOH}$ в присутствии катализатора, исследован методом твердотельного ядерного магнитного резонанса ${ }^{1} \mathrm{H}$ и ${ }^{13} \mathrm{C}$. По сигналу ${ }^{1} \mathrm{H}$ сделано заключение о возможных особенностях строения оболочки молекулы. В спектре ${ }^{13} \mathrm{C}$ обнаружено сохраняющееся с повышением температуры расщепление пика, которое, согласно предположению, обусловлено наличием изомеров фуллеренола.

Работа выполнена при частичной финансовой поддержке Программы повышения конкурентоспособности ФГАОУ ВО СПбПУ „5-100-2020“.

DOI: 10.21883/FTT.2018.07.46138.018

Среди многочисленных углеродных структур, которые в перспективе можно было бы использовать на практике, большое внимание привлекают водорастворимые формы фуллеренов - полигидроксилированные фуллерены (фуллеренолы), [1]. Данные соединения имеют общую формулу $\mathrm{C}_{60}(\mathrm{OH})_{n}$, где величина $n$ может изменяться в зависимости от условий получения, а модификация поверхности молекул $\mathrm{C}_{60} \mathrm{OH}$-группами обеспечивает их высокую способность к растворению в воде (последнее особенно интересно в связи с биомедицинскими задачами, [2]). Известно, однако, что эти вещества чувствительны к методикам синтеза, которые могут оказывать определенное влияние на детали их строения. Целью настоящей работы было выяснение того, какие особенности имеют спектры ядерного магнитного резонанса (ЯМР) образцов, полученных по обеспечивающей высокую чистоту продукта технологии, описанной ниже.

Материал синтезировался в два этапа, по схеме, рассмотренной в $[3,4]$. Вначале эрозией графитовых электродов в электрической дуге в атмосфере гелия в условиях низкотемпературной плазмы была получена фуллеренсодержащая сажа. Из нее с помощью ксилола выделялась смесь фуллеренов, из которой на хроматографической колонке проводилось отделение $\mathrm{C}_{60}$. Очистка $\mathrm{C}_{60}$ выполнялась путем многократного пропускания раствора, обогащенного данным фуллереном, через колонку с углеродным сорбентом, а содержание $\mathrm{C}_{60}$ в каждой фракции контролировалось методом высокоэффективной жидкостной хроматографии. После многоступенчатой очистки был получен фуллерен $\mathrm{C}_{60}$ с чистотой 99.9 wt.\%, без примесей $\mathrm{C}_{70}$ и высших фуллеренов, и содержанием оксида $\mathrm{C}_{60} \mathrm{O}$ менее $0.1 \%$. Для синтеза фуллеренола использовались бензол, гидроксид натрия, и, в качестве межфазного катализатора, раствор гидроксида тетрабутиламмония с концентрацией 20 wt.\%. В отфильтрованный бензольный раствор $\mathrm{C}_{60}$ при интенсивном перемешивании добавлялся концентрированный (45 wt.\%) раствор $\mathrm{NaOH}$, и раствор гидроксида тетрабутиламмония. После полного удаления щелочи фуллеренол высушивался в вакууме при $40^{\circ} \mathrm{C}$. По результатам комплексного термического и элементного анализа образца, и с учетом того, что в нем, по оценке, содержится 4.8\% гидратной воды, установлено, что полученному соединению наиболее точно соответствует формула $\mathrm{C}_{60}(\mathrm{OH})_{42} \cdot 4 \mathrm{H}_{2} \mathrm{O}$.

Для характеризации синтезированного вещества использовались рентгеновская фотоэлектронная и инфракрасная спектроскопия. Применение первого из этих методов подробно описано в [5], где приведены результаты, показывающие, что фотоэлектронный спектр нашего образца может быть разложен на компоненты, соответствующие связям углерод-углерод и углеродгидроксильная группа при соотношении этих составляющих $3: 1$. Оптическая методика также подтвердила, что полученное соединение представляет собой фуллеренол. Его инфракрасный (ИК) спектр показан на рис. 1. Видно, что там присутствуют характерные для фуллеренолов пики поглощения при волновых числах $\tilde{v}$, равных 


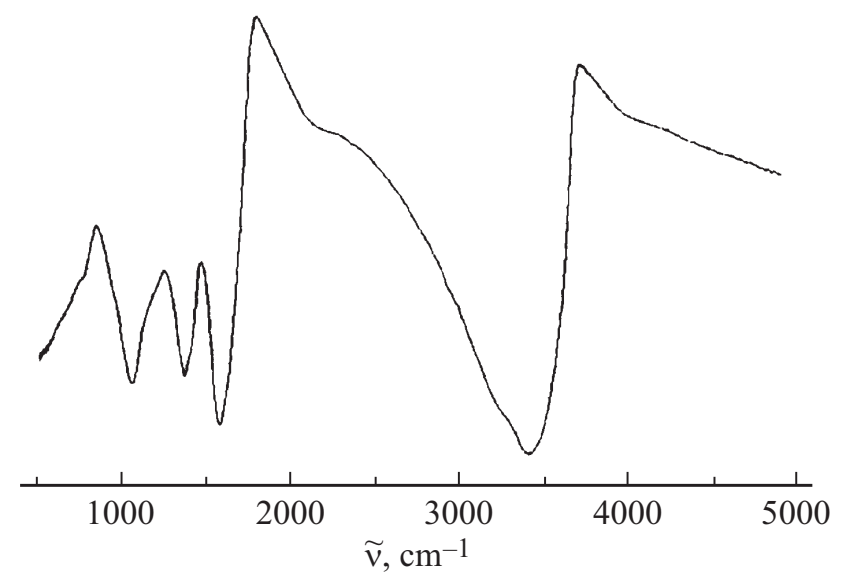

Рис. 1. ИК-спектр исследованного образца.

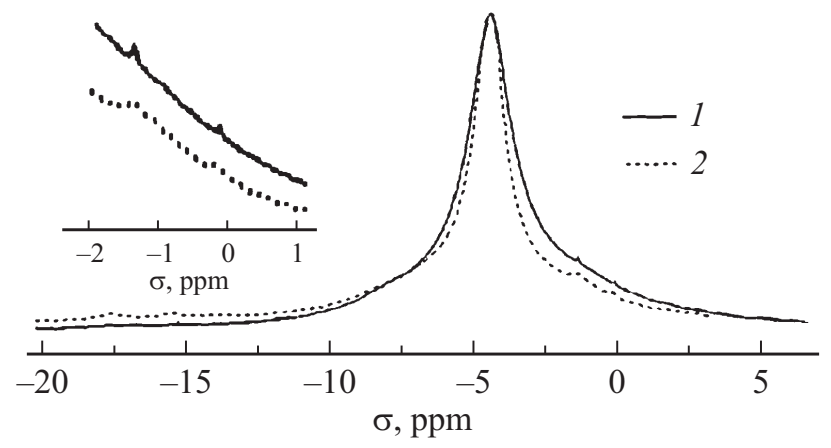

Pис. 2. Спектр ЯМР ${ }^{1} \mathrm{H}$, полученный при $T=25^{\circ} \mathrm{C}$ и разных частотах вращения: $1-10 \mathrm{kHz}, 2-20 \mathrm{kHz}$. На вставке: низкополевая область химических сдвигов в увеличенном масштабе.

$1080 \mathrm{~cm}^{-1}, 1379 \mathrm{~cm}^{-1}, 1620 \mathrm{~cm}^{-1}$ и $3400 \mathrm{~cm}^{-1}$, которые отвечают валентным колебаниям связей $\mathrm{C}-\mathrm{O}$, деформационным колебаниям симметричных связей $\mathrm{C}-\mathrm{O}-\mathrm{H}$, и валентным колебаниям связей $\mathrm{C}-\mathrm{C}$ и $\mathrm{O}-\mathrm{H}$ соответственно, [6-8]. Нагрев образца до $90^{\circ} \mathrm{C}$ вид спектра не изменял.

Спектры ЯМР наблюдались на радиоспектрометре Bruker Avance III WB 400, оснащенном датчиком с системами вращения образцов под магическим углом и температурной стабилизации. Для ядер ${ }^{13} \mathrm{C}$ рабочая частота составляла $100.613 \mathrm{MHz}$. Частота вращения ротора могла регулироваться, в наших экспериментах она составляла 10 или $20 \mathrm{kHz}$. При определении химических сдвигов $\sigma$ в качестве внешнего стандарта использовался тетраметилсилан $(\sigma=0 \mathrm{ppm})$. Для возбуждения сигналов ЯМР применялась одноимпульсная последовательность с периодом повторения 5s и длительностью импульса $2.6 \mu \mathrm{s}$. В процессе регистрации сигналов могла использоваться кросс-поляризационная методика CP/MAS. Измерения были выполнены при температурах $T$ от комнатной до $90^{\circ} \mathrm{C}$.

На рис. 2 показаны спектры ЯМР протонов, полученные на двух частотах вращения при $T=25^{\circ} \mathrm{C}$. Уве- личение частоты, как это видно из рисунка, повышает разрешение метода и облегчает регистрацию отдельных линий.

Наиболее интенсивный сигнал ${ }^{1} \mathrm{H}$ наблюдался при химическом сдвиге $4.4 \mathrm{ppm}$, но при этом отклик существовал также в низко- и высокополевой областях спектра. Сдвиг в сторону больших отрицательных значений $\sigma$ частично связан с аппаратурными эффектами, проявляющимися при слабых сигналах, в то время как резонансы, имеющие $\sigma>2 \mathrm{ppm}$ (см. вставку на рис. 2) могут быть отнесены к протонам ОН-групп, а $\sigma<4 \mathrm{ppm}$ - к ароматическим протонам, [9]. По сравнению с результатом, полученным в [9], в нашем случае обнаружено уширение основного пика, что не позволило наблюдать отдельные линии при сдвигах, незначительно превышающих 5 ppm, хотя в этой области заметно присутствие очень слабо разрешенного сигнала с $\sigma \approx 8 \mathrm{ppm}$. Кроме того, на спектре ЯМР ядер ${ }^{1} \mathrm{H}$ можно видеть слабоинтенсивные линии с химическими сдвигами около 15.3 и $17.5 \mathrm{ppm}$, которые могут соответствовать различным окисленным структурным производным. Отличие наших данных от результатов [9] состоит, прежде всего, в увеличенной ширине пиков, что, по всей видимости, связано с бо́льшим количеством гидроксильных групп $n$. При этом в положении основных резонансов наблюдается корреляция, что указывает на такое строение гидратной оболочки, при котором ОН-группы находятся в непосредственном взаимодействии с фуллереновым каркасом.

Приведенный на рис. 3 спектр сигнала ЯМР ${ }^{13} \mathrm{C}$ $(10 \mathrm{kHz})$ в целом соответствует данным других работ $[6,8,9]$. В отличие от сигнала чистого фуллерена он имеет несколько пиков, обусловленных существованием различных связей углерода с присоединенными группами (их соотнесение можно найти, например, в [8]). Особенность наших результатов состоит в том, что пик с химическим сдвигом около 165 ppm, который приписан одновалентной группе $\mathrm{O}=\mathrm{C}-\mathrm{O}$ (карбоксил), оказывается расщепленным на две близко расположенных линии, причем этот эффект воспроизводится при выполнении эксперимента в разных условиях.

На рис. 4 приведены спектры ЯМР ${ }^{13} \mathrm{C}$, полученные на частоте вращения $20 \mathrm{kHz}$ при различных темпе-

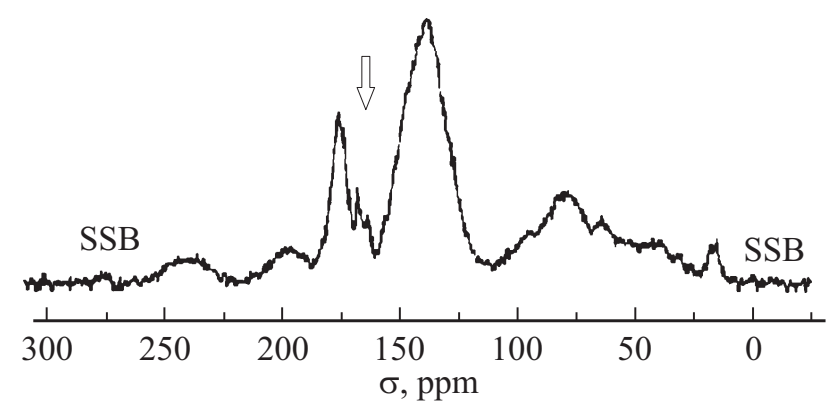

Рис. 3. Спектр ЯМР ${ }^{13} \mathrm{C}$, снятый при частоте вращения $10 \mathrm{kHz}$ и температуре $25^{\circ} \mathrm{C}$ (стрелкой отмечен расщепленный пик, SSB - вращательные артефакты). 

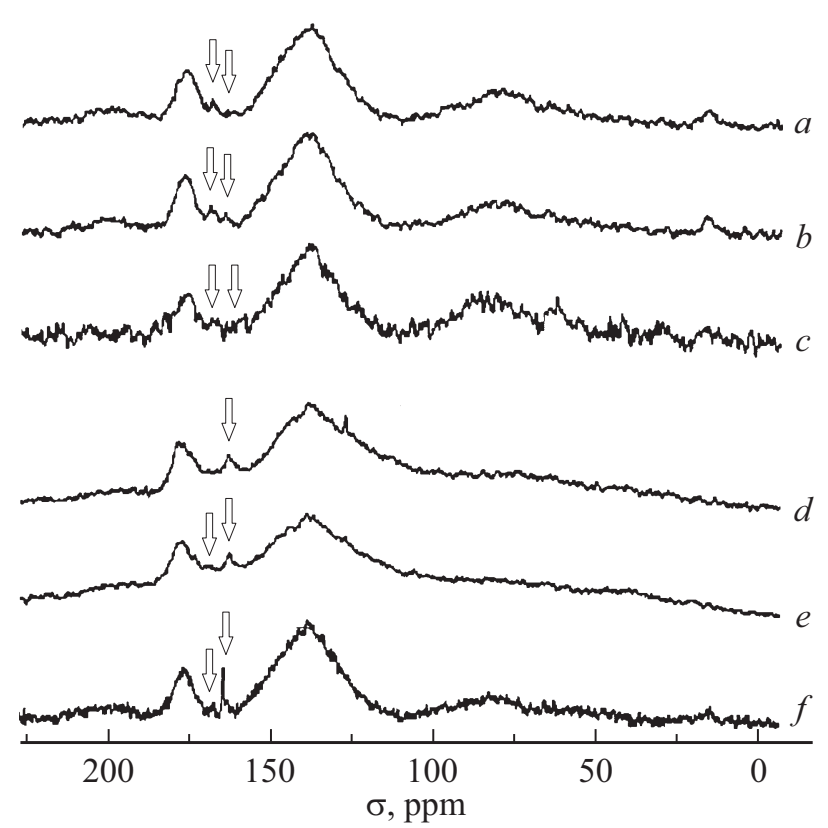

Pис. 4. Спектры ЯМР ${ }^{13} \mathrm{C}$, полученные на частоте вращения $20 \mathrm{kHz}$ при нагреве и последующем охлаждении образца, $a-T=30^{\circ} \mathrm{C}, b-T=50^{\circ} \mathrm{C}, c-T=70^{\circ} \mathrm{C}$ (СР отключена), $d-T=90^{\circ} \mathrm{C}, e-T=30^{\circ} \mathrm{C}, f-T=30^{\circ} \mathrm{C}$ (СР отключена). Стрелками отмечены компоненты расщепленного пика.

ратурах (видно, что увеличение частоты приводит к уходу связанных с вращением пиков SSB за пределы обычных для органических соединений значений химических сдвигов). Рис. $3, a-d$ показывают, что нагрев не оказывает существенного влияния на положение компонентов расщепленного пика. Отношение сигнал/шум для спектров, полученных методом CP/MAS, с увеличением температуры падает, и при больших $T$ для их получения методика прямого возбуждения оказывается более эффективной. Это может быть связано с тем, что при нагревании образца молекулы воды отрываются от поверхности фуллеренола, нарушая условия переноса намагниченности от протонов к ядрам углерода. Тем не менее, даже при включенной кросс-поляризации видно, что при $T=90^{\circ} \mathrm{C}$ один из сигналов от карбоксильных групп становится очень слабым (рис. 3, $d$ ). Понижение температуры (рис. 3,e-f) восстанавливает обе компоненты, причем в ходе цикла „нагрев-охлаждение“ обнаруживается эффект, внешне проявляющийся как изменение интенсивности одной компоненты за счет другой.

Последнее обстоятельство могло бы указывать на температурное изменение равновесия между кетонными и енольными формами, смесью которых, по данным [6], в общем случае является полигидроксилированный фуллерен. Однако нами характерная для кетонов [8] линия поглощения в ИК-спектре при $\tilde{v}=1720 \mathrm{~cm}^{-1}$ не наблюдалась (рис. 1), причем сам спектр, как уже говорилось выше, не изменялся с повышением температуры. Следовательно, для объяснения появления двух указан- ных линий следует привлечь способность системы к образованию других изомеров. Сам по себе углеродный фуллереновый каркас способен к структурной изменчивости [10], и не исключено, что она дает определенный вклад в наблюдавшееся расщепление. Следует учесть, однако, обнаруженную особенность температурного поведения спектров ЯМР, предполагающую известную лабильность изомерных форм, которая больше отвечает природе гидратной оболочки фуллеренола. Присоединение ОН-групп к центральной молекуле $\mathrm{C}_{60}$ с образованием карбоксила возможно несколькими способами [8], что и лежит, по нашему предположению, в основе образования зарегистрированных изомеров. (Отметим, что эксперимент обнаруживает их в приблизительно равном количестве.)

Таким образом, нами продемонстрировано, что использование описанной выше технологии обеспечивает получение фуллеренола, в котором образующие его изомеры обладают различимыми физическими свойствами, т.е. показана технологическая управляемость продуктом синтеза, а также выдвинуто общее предположение о его строении, учитывающее особенности спектров ЯМР.

Авторы благодарят ресурсный центр СПбГУ за предоставление аппаратуры для проведения экспериментов и Ю.М. Жукова за характеризацию образцов методом рентгеновской фотоэлектронной спектроскопии.

\section{Список литературы}

[1] G.V. Andrievsky, V.K. Klochkov, A.B. Bordyuh, G.I. Dovbeshko. Chem. Phys. Lett. 364, 8 (2002).

[2] С.М. Андреев, Е.Н. Башкатова, Д.Д. Пургина, Н.Н. Шершакова, М.Р. Хаитов. Иммунология 36, 1, 67 (2015).

[3] J. Li, A. Takeuchi, M. Ozawa, X. Li, K. Saigo, K. Kitazawa. J. Chem. Soc. Chem. Commun. 23, 1784 (1993).

[4] К.Н. Семенов, Д.Г. Летенко, Н.А. Чарыков, В.А. Никитин, М.Ю. Матузенко, В.А. Кескинов, В.Н. Постнов, А.А. Копырин. ЖПХ 83, 1948 (2010).

[5] А.Е. Карпунин, Ю.М. Жуков, В.И. Герасимов, О.В. Проскурина, И.В. Плешаков, А.А. Нечитайлов, Н.В. Глебова. Материалы XIV Междунар. конф. „Физика диэлектриков“ (Диэлектрики-2017), СПб 2, 96 (2017).

[6] L.Y. Chiang, R.B. Upasani, J.W. Swirczewski. J. Am. Chem. Soc. 115, 5453 (1993).

[7] G.M. Xing, J. Zhang, Y.L. Zhao, J. Tang, B. Zhang, X.F. Gao, H. Yuan, L. Qu, W.B. Cao, Z.F. Chai, K. Ibrahim, R. Su. J. Phys. Chem. B 108, 11473 (2004).

[8] Zhenzhen Wang, Xueling Chang, Zhanghui Lu, Min Gu, Yuliang Zhao, Xingfa Gao. Chem. Sci. 5, 2940 (2014).

[9] D.V. Andreeva, O.V. Ratnikova, E.Yu. Melenevskaya, A.V. Gribanov. Int. J. Polymer Anal. Character. 12, 105 (2007).

[10] V.I. Gerasimov, A. Trofimov, O. Proskurina. Mater. Phys. Mech. 20, 25 (2014).

Редактор К.В. Емцев 